\title{
Trends in fall-related ambulance use and hospitalisation among older adults in NSW, 2006- 2013: a retrospective population-based study
}

\section{Serene S Paula,b,j, Lara Harveyc ${ }^{c}$, Therese Carrolld ${ }^{d}$, Qiang Lie, Soufiane Boufous ${ }^{\dagger}$, Annabel Priddis ${ }^{d}$, Anne Tiedemanna, Lindy Clemson ${ }^{b}$, Stephen R Lordc, Sandy Mueckes, Jacqueline CT Close ${ }^{\mathrm{ch}}$, Serigne Lo' and Catherine Sherrington ${ }^{2}$}

${ }^{a}$ Musculoskeletal Health Sydney, School of Public Health, Sydney Medical School, University of Sydney, NSW, Australia

b Faculty of Health Sciences, University of Sydney, NSW, Australia

- Neuroscience Research Australia, UNSW Sydney

${ }^{d}$ Centre for Population Health, NSW Ministry of Health, Sydney, Australia

\begin{abstract}
e The George Institute for Global Health, Sydney, NSW, Australia ${ }^{\dagger}$ Transport and Road Safety Research, UNSW Sydney, Australia 9 NSW Ambulance, Sydney, Australia

h Prince of Wales Clinical School, UNSW Sydney, Australia

' Melanoma Institute Australia, Sydney, NSW

i Corresponding author: serene.paul@sydney.edu.au
\end{abstract}

\section{Article history}

Publication date: October 2017 Citation: Paul SS, Harvey L, Carroll T, Li $\mathrm{Q}$, Boufous S, Priddis A, et al. Trends in fall-related ambulance use and hospitalisation among older adults in NSW, 2006-2013: a retrospective population-based study. Public Health Res Pract. 2017;27(4):e27341701 https://doi.org/10.17061/phrp27341701

\section{Key points}

- Fall-related ambulance service use has increased over time

- Fall prevention interventions should include safety and avoidance strategies

- Fall management strategies should continue to be targeted at those aged older than 85 in an effort to reduce their fall-related health service use

- There is a need for specific fall injury prevention strategies in aged care facilities

\section{Abstract}

Objective and importance of study: To describe characteristics and temporal trends of fall-related ambulance service use and hospital admission in older adults in New South Wales (NSW), Australia. Such information will facilitate a more targeted approach to planning and delivery of health services to prevent falls and their adverse sequelae in different groups of older adults.

Study type: Retrospective population-based descriptive study.

Methods: Fall-related ambulance use and hospital admissions for all falls and injurious falls in NSW residents aged $\geq 65$ years between 2006 and 2013 were obtained from two discrete sources of routinely collected data. Rates of use are presented descriptively.

Results: There were 314041 occasions of fall-related ambulance use by older adults and 331311 fall-related hospitalisations, of which $69 \%$ ( $n=227$ 753) were for injurious falls. Fractures accounted for $57 \%$ of injurious hospitalisations. Slips and trips were the most common mechanism of falls requiring hospitalisation (52\%). Residents of aged care facilities had a greater proportion of fall injury hospitalisations compared with people living in the community (85\% and 65\%, respectively).

Conclusions: Rates of fall-related ambulance use and hospitalisation were similar and continued to increase over time. Increased effort is needed to prevent falls and associated injury among older people in NSW, particularly among people living in aged care facilities. Ongoing monitoring of rates and the characteristics of people who fall are needed to determine the long-term impact of fall prevention interventions. 


\section{Introduction}

Each year, one-third of people aged $\geq 65$ years fall, particularly older females. ${ }^{1}$ Fall prevention strategies aim to address this problem, but have yet to affect the continued increase in fall-related health service use among older adults. ${ }^{2}$

Older people who have had a fall comprise a substantial proportion of users of paramedic and hospital services. Falls in older adults constitute $5 \%$ of emergency ambulance responses in Australia $^{3}$, and $66-76 \%$ of older people who have had a fall require transport to hospital. ${ }^{3}$ Of all falls in older adults, $10-15 \%$ result in fracture ${ }^{4,5}$, a further $15-20 \%$ result in other serious injuries requiring medical attention $n^{4,6}$ and approximately $5 \%$ result in injury warranting hospital admission. ${ }^{6}$ Despite the small proportion of falls resulting in hospitalisation, the costs associated with these admissions account for more than $80 \%$ of the cost of treatment for fall-related injuries. ${ }^{6}$ Because the proportion of older adults in the population is increasing, it is unsurprising that fall-related hospitalisations are increasing in New South Wales (NSW), Australia. ${ }^{7}$

The studies cited above provide a clear understanding that falls in older adults remain problematic, and that people who fall use substantial healthcare resources. ${ }^{8}$ However, there has not previously been a systematic investigation about patterns of fall-related ambulance use over time, and few studies of fall-related hospitalisations have identified the characteristics of individuals with multiple admissions. Understanding recent patterns of serious falls that require health service use (such as multiple admissions to hospital or paramedic attendance) in older adults at a population level, along with these individuals' characteristics, will facilitate a more targeted approach to planning and delivery of health services to prevent falls and their adverse sequelae in different groups of older adults.

For the NSW population aged $\geq 65$ years, this study aims to: 1) describe fall-related ambulance service use, and fall-related and fall injury-related hospital admission; 2) explore patterns of service use by age, sex and injury type; and 3) describe rates of service use over time, by age groups and sex.

\section{Methods}

Two data sources, NSW Ambulance data and the NSW Admitted Patient Data Collection (APDC), were used to identify people aged 65 years and older who: 1) requested an ambulance for a fall-related incident; or 2) were admitted to a hospital in NSW between 1 January 2006 and 31 December 2013. Interstate residents were excluded from both datasets.

Ethics approval for this study was granted by the NSW Population and Health Services Research Ethics Committee. This study conforms to STROBE guidelines.

\section{Ambulance records}

Ambulance use data were obtained from routinely collected NSW Ambulance Computer Aided Dispatch (CAD), Patient Health Care Record (PHCR) and electronic Medical Record (eMR) databases. NSW Ambulance is the sole provider of emergency out-of-hospital care in NSW. CAD data gathered during the emergency call includes information about the problem and the patient's location. PHCR and eMR datasets contain patient-related clinical information recorded by attending paramedics. Use of eMR was introduced in a staged approach from 2011. Both systems remain in use, because not all paramedics have access to the eMR system at all times. NSW Ambulance routinely links CAD and clinical records for each patient.

Following a patient assessment, not all patients are transported to an emergency department (ED) by paramedics. Referral pathways have been established since 2011 so that, at the time of the patient encounter, paramedics can recommend subsequent review by community-based healthcare providers. Additionally, since 2008, NSW Ambulance has implemented an extended care paramedic role, where experienced paramedics receive additional education to treat patients with low-acuity conditions at the scene.

In the NSW Ambulance data collections, eligible records were those where a 'falls' problem was recorded in the CAD database for patients aged $\geq 65$ years, as documented in their clinical record. The master incident number was used to deduplicate records so that a single ambulance dispatch was used in analyses. Transport disposition was used to exclude ineligible records ('operational standby', 'PHCR incident cancel en route', 'PHCR not required', 'unable to locate patient', 'assist/treat/load'), and to identify records transported ('transported') or not transported (all other dispositions including 'deceased on examination') to hospital.

For CAD 'falls' records, information about associated injuries was obtained from the corresponding clinical record completed by paramedics. NSW Ambulance paramedics classify and document care using clinical protocols (trauma, cardiac/cardiovascular, drug/ toxicology, environment/envenomation, medical/ surgical, and specialty care such as obstetrics or mental health). Patients with 'limb injuries and fractures' and 'limb realignment and difficult extraction' were grouped as limb fractures; 'soft tissue injuries to the face and neck', 'epistaxis' and 'wound care' were grouped as soft tissue injury; 'head injury', 'spinal injuries', 'chest injuries', 'penetrating trauma', 'pelvic injuries' and 'prehospital management of serious trauma or deteriorating trauma patient' were grouped as serious injury; with the remaining injuries grouped as other injuries. Protocols describing medical conditions were grouped as noninjuries; and protocols without specific clinical diagnoses were grouped as unspecified. Because an individual may have requested more than one ambulance over the study 
period, ambulance use results are presented as 'records' rather than 'patients'.

\section{Hospitalisations}

The APDC records all public and private hospitalisations that occur in NSW. These episodes of care end with discharge, transfer to another facility, death, or when the service category changes (e.g. a change from acute to subacute care within the same facility), so an individual may have multiple episodes of care recorded in the APDC for a single hospital admission. Using probabilistic matching of individuals' names, date of birth and address with ChoiceMaker software, the NSW Centre for Health Record Linkage internally linked the APDC to itself to identify all episodes of care that constituted the overall hospital stay for each patient and to determine the number of individual patients admitted to hospital.

Data about patient diagnoses were coded using the International Classification of Diseases and Related Health Problems, 10th revision, Australian Modification (ICD-10-AM). ${ }^{9}$ Fall-related hospitalisations were determined using external cause codes W00-W19. Injurious falls were subsequently identified using primary diagnosis codes S00-T75 and T79, and described in categories similar to those described in the NSW Ambulance data, except that upper limb, lower limb and hip fractures are singularly described in NSW Ambulance data as 'limb fractures'. Records with a place of occurrence 'Y92.14' or source of referral ' 6 - Nursing Homes' were used to estimate the number of residents of aged care facilities who were hospitalised because of a fall. To avoid overestimating the number of hospital admissions per fall, we excluded episodes of care coded as statistical transfers (i.e. changes in the service category or transfers between different units within the same hospital); and transfers and/or discharges to a different hospital, residential aged care facility or ED, or to ambulatory care.

\section{Statistical analyses}

The characteristics of older people who used ambulance services or were admitted to hospital because of a fall (including residents of aged care facilities) and the circumstances of the fall-related incident (including fall-related injuries) were summarised using descriptive statistics. Geographical remoteness was estimated for each location (Statistical Local Area 2011 [SLA2011]) using rounded scores of the 2011 Accessibility/ Remoteness Index of Australia ${ }^{10}$; low scores indicate low remoteness. Socio-economic status was obtained by mapping the Australian Bureau of Statistics (ABS) 2011 socio-economic indices of relative socio-economic advantage and disadvantage (cat. no. 2033.0.55) to SLA-2011'11; low scores indicate a low proportion of advantage and a high proportion of disadvantage. Rates (per 100000 people) of fall-related ambulance use and hospitalisations for all falls and injurious falls were calculated as: 1) directly age standardised to the estimated resident Australian population at 30 June 200111; and 2) crude rates based on the NSW population aged $\geq 65$ years, using annual estimates from the ABS for each year of the study. ${ }^{11}$ SAS Enterprise Guide v5.1 (SAS Institute Inc, Cary NC) was used for analysis.

\section{Results}

In NSW from 2006 to 2013, there were 314041 records of fall-related ambulance use by older adults and 331311 fall-related hospitalisations (supplementary Table S1 http://hdl.handle.net/2123/16249), of which 69\% (227 753 admissions) were for injurious falls. These admissions comprised 200016 individuals, and $84 \%$ of these individuals (166 978) were admitted to hospital for injury associated with the fall (Table S2 http://hdl.handle. net/2123/16249).

People who used ambulance services and were admitted to hospital for falls were similar in terms of age, sex, socio-economic status and remoteness index (Table S1 http://hdl.handle.net/2123/16249). Fall-related health service use was highest among females and lowest among adults aged 65-74 years.

Of all falls attended by paramedics, $74 \%$ were transported to hospital (Table S3a http://hdl.handle. net/2123/16249) and 46\% were associated with an injury. The largest proportion of associated injuries was limb fractures (30\%). Serious trauma accounted for $15 \%$ of injuries. Most people with injury were transported to hospital (85-94\%), particularly for fractures or serious injury (93-94\%). Injuries appeared to be evenly spread across all ages. The reason for falling was not specified in $35 \%$ of ambulance records.

Females of all ages had higher rates of hospitalisation for injurious falls (Figure S1c http://hdl.handle. net/2123/16249), particularly for upper and lower limb fractures, including hip fractures (Table S3b http://hdl.handle.net/2123/16249). Non-injurious falls involving medical issues (e.g. nausea, urinary tract infection or a neurological condition) accounted for $31 \%$ of fall-related hospitalisations. Overall, adults aged 65-74 years had fewer fall-injury admissions, but more admissions for upper and lower limb fractures excluding hip fractures. People aged $\geq 85$ years had more admissions for hip fractures, soft tissue and nonspecified injuries

Most adults had a single fall-related hospitalisation (69\%), 20\% were admitted twice and $1 \%$ had frequent $(\geq 10)$ fall-related hospitalisations (Table S2 http://hdl.handle.net/2123/16249). A greater proportion of females, across all age groups, had multiple fallrelated hospital admissions (Table S4 http://hdl.handle. net/2123/16249). Fewer adults aged 65-74 years, and more people aged $\geq 85$ years during their first fall separation, had multiple fall-related admissions. At the first fall separation, there were no obvious differences 
in injuries sustained or residential aged care status between people with single or multiple admissions. Most fall admissions by residents of aged care facilities were in females and in people aged $\geq 85$ years (Table S5 http://hdl.handle.net/2123/16249). Residents of aged care facilities had more injurious falls admissions (86\% compared with $65 \%$ in people living in the community), particularly fractures (46\% versus 38\%, respectively).

The cause of the fall was not specified in $29 \%$ of fall-related hospitalisations overall, and was not specified by $43 \%$ of people aged $\geq 85$ years (Table S6 http://hdl.handle.net/2123/16249). Where the cause of the fall could be identified, most occurred because of slips and trips (52\%). Falling off ladders and buildings (2\% of falls) occurred mostly in males (67-78\%), particularly in those aged 65-74 years.

Age-standardised rates of fall-related health service use in 2013 per 100000 people were 3814 for ambulance, 4167 for hospitalisation and 2791 for injurious hospitalisations. These rates increased over time (Table 1). Crude rates of fall-related health service use were greater in females and with increasing age (Table 1; Figures S1a-c http://hdl.handle.net/2123/16249).

\section{Discussion}

Fall-related health service use (i.e. ambulance use and hospitalisations) among older adults in NSW increased between 2006 and 2013. These rates were higher than reports from earlier years ${ }^{8}$ but similar to figures from recent years. ${ }^{7}$ Fall-related health service use was highest among females and people aged $\geq 85$ years. ${ }^{1,3,7,8}$ The latter likely reflects the increasing frailty and accumulation of fall risk factors with increasing age that contribute to

Table 1. Age-standardised and crude rates (per 100000 people) of fall-related ambulance use, fall-related and fall injury-related hospital separations in NSW, by sex and age groups, 2006-2013

\begin{tabular}{|c|c|c|c|c|c|c|c|c|}
\hline \multirow[b]{2}{*}{ Category } & \multirow[b]{2}{*}{ Year } & \multirow{2}{*}{$\begin{array}{c}\text { Age-standardised } \\
\text { rate (all) }\end{array}$} & \multicolumn{6}{|c|}{ Crude rate } \\
\hline & & & All & Females & Males & 65-74 years & 75-84 years & $85+$ years \\
\hline \multirow{8}{*}{$\begin{array}{l}\text { NSW Ambulance } \\
\text { dispatches }\end{array}$} & 2006 & 3220 & 3359 & 3759 & 2837 & 1260 & 4114 & 10114 \\
\hline & 2007 & 3452 & 3627 & 4091 & 3041 & 1336 & 4397 & 10961 \\
\hline & 2008 & 3610 & 3804 & 4265 & 3228 & 1423 & 4581 & 11394 \\
\hline & 2009 & 3740 & 3946 & 4417 & 3366 & 1458 & 4674 & 12120 \\
\hline & 2010 & 3768 & 3987 & 4507 & 3357 & 1422 & 4706 & 12444 \\
\hline & 2011 & 3964 & 4198 & 4717 & 3574 & 1533 & 4841 & 13264 \\
\hline & 2012 & 3895 & 4102 & 4647 & 3457 & 1483 & 4806 & 12994 \\
\hline & 2013 & 3814 & 4009 & 4556 & 3370 & 1461 & 4667 & 12803 \\
\hline \multirow{8}{*}{$\begin{array}{l}\text { Fall-related hospital } \\
\text { admissions }\end{array}$} & 2006 & 3498 & 3654 & 4496 & 2612 & 1358 & 4341 & 11454 \\
\hline & 2007 & 3553 & 3739 & 4572 & 2716 & 1396 & 4363 & 11702 \\
\hline & 2008 & 3567 & 3760 & 4648 & 2677 & 1435 & 4453 & 11345 \\
\hline & 2009 & 3799 & 4010 & 4870 & 2973 & 1507 & 4677 & 12399 \\
\hline & 2010 & 4044 & 4273 & 5160 & 3214 & 1635 & 4958 & 13111 \\
\hline & 2011 & 4260 & 4499 & 5499 & 3316 & 1766 & 5175 & 13752 \\
\hline & 2012 & 4180 & 4396 & 5305 & 3329 & 1708 & 5072 & 13633 \\
\hline & 2013 & 4167 & 4371 & 5149 & 3466 & 1744 & 5015 & 13515 \\
\hline \multirow{8}{*}{$\begin{array}{l}\text { Fall injury-related } \\
\text { hospital admissions }\end{array}$} & 2006 & 2544 & 2660 & 3360 & 1794 & 982 & 3062 & 8660 \\
\hline & 2007 & 2542 & 2681 & 3374 & 1830 & 981 & 3031 & 8751 \\
\hline & 2008 & 2561 & 2709 & 3455 & 1800 & 1005 & 3081 & 8638 \\
\hline & 2009 & 2608 & 2761 & 3506 & 1863 & 1035 & 3102 & 8860 \\
\hline & 2010 & 2723 & 2888 & 3634 & 1997 & 1070 & 3268 & 9214 \\
\hline & 2011 & 2776 & 2948 & 3691 & 2070 & 1094 & 3282 & 9533 \\
\hline & 2012 & 2782 & 2939 & 3692 & 2057 & 1098 & 3292 & 9532 \\
\hline & 2013 & 2791 & 2941 & 3634 & 2135 & 1115 & 3302 & 9498 \\
\hline
\end{tabular}


increased fall incidence ${ }^{12}$, and highlights the importance of targeting fall prevention interventions in this group.

Rates of fall-related ambulance use were slightly lower than fall-related hospital admissions. The sex-related difference in rates of fall-related ambulance use was also smaller than for hospital admissions, and lower than previous reports of ambulance use by older females who had a fall. ${ }^{3}$ Most (74\%) fall events in older adults attended by paramedics resulted in the person being transported to hospital ${ }^{13,14}$, although transportation rates were higher in instances where a fall led to an injury (85-94\%). It may be that some people who sustained minor or no fall-related injury required 'lift only' assistance ${ }^{15}$ or were managed in the community. ${ }^{16}$ As paramedic-initiated community-based referral pathways expand in NSW, it is anticipated that transportation rates to EDs will decline, particularly for non-injurious falls. It was not possible to accurately ascertain the reasons for nontransportation to an ED in our data. Some of those initially not transported to hospital may have refused transport at that time. ${ }^{16}$ Those not initially transported may have later presented to an ED by alternative modes of transport (such as private car) or requested a subsequent paramedic attendance. ${ }^{16}$ Not all patients with fractures or serious injury were transported to hospital (6-7\%, including those who died at the scene of the fall).

For most fall circumstances, females and the older age groups accounted for the largest proportions within each category (e.g. falls due to trips and slips, or falls in the toilet/shower). The notable difference was in injuries from falling off ladders or scaffolding, or from diving, which were more prevalent in males aged 65-74 years. ${ }^{17}$ It appears that men continue to take risks as they age: 'younger old' males account for the majority of trauma and hospital admissions following falls from ladders. ${ }^{17}$ Fall prevention and health promotion interventions need to account for increased risk taking by older men to promote both safe use and avoidance messages.

Two-thirds of fall-related hospitalisations were for fall-related injuries ${ }^{3,7}$, although less than $50 \%$ of falls in the ambulance data were recorded as injurious. Injuries documented in ambulance clinical records are provisional, paramedic-determined diagnoses. The exact nature of injuries and determination of injury severity require confirmation by radiologic imaging or other assessments in hospital. Residents of aged care facilities also had a high proportion of injurious fall hospitalisations, although, unlike other studies, we did not find that males in residential aged care facilities had a higher incidence of falls and fall injuries. ${ }^{18,19}$ However, we were unable to accurately determine the number of aged care facility residents in our dataset because ED and Australian Government residential aged care data were not included. ${ }^{20}$ Females had higher instances of fracture than males ${ }^{8}$, particularly upper limb fractures (81\% and $74 \%$, respectively, for all fractures). ${ }^{21}$ People with multiple hospital separations had a higher proportion of non-injurious falls, although this was not apparent during their first admission. This may be because of the higher fall risk for some groups, such as those with neurological conditions. ${ }^{22}$ Future research is warranted to determine which older people who have had a fall have a high likelihood of readmission, the conditions associated with multiple admissions and the average time between readmissions. Demonstrated age and sex differences in the types of injuries and circumstances of falls suggest that it may be beneficial to classify people who have had a fall according to specific risk profiles. This may assist in the targeted delivery of content-specific fall prevention and health promotion interventions ${ }^{5,23}$, and limit future hospitalisations.

This study has several limitations and strengths. The methodology did not permit patients' entire healthcare journeys to be examined because APDC data were not linked to NSW Ambulance records or ED data. Linkage would be beneficial in understanding the proportion of falls admissions that arrived by ambulance; the proportion of people who were not initially transported to an ED by ambulance but were subsequently admitted, or who arrived by any mode of transport but were discharged from the ED; and the characteristics of older people who have had a fall who use ambulance and ED services multiple times a year. Linkage with death records would also provide more comprehensive information. Nevertheless, it is likely that both datasets represented a similar population of older adults in NSW.

Coding differences between NSW Ambulance and the APDC made it difficult to compare the patterns of injury sustained by older people who had a fall. A small underestimation of fall-related health service use in NSW may have occurred because older adults living near state borders may have been attended by other ambulance services or been hospitalised interstate; there was a lack of ED data; and only $72 \%$ of actual falls records are captured by the CAD system. ${ }^{13,14}$ Using the CAD database to identify a population of interest is problematic because information collected at the time of the emergency call may not accurately reflect the true nature of the patient's problems. The caller may be a third party and, at times of anxiety, information provided to calltakers may be imprecise. Routinely collected information is not primarily collected for research purposes, as noted in missing data across various outcomes and lack of detail in coding of some variables (e.g. a large proportion of unspecified fall circumstances in hospitalised patients). Greater variability in APDC coding of external cause codes to identify falls compared with greater accuracy of coding of primary diagnosis codes to identify injury ${ }^{24}$ may have magnified the differences in rates between admissions for all falls compared with injurious falls.

Key strengths of this paper are the reported patterns of fall-related ambulance use over time and identification of individual patients within the APDC dataset, which enable quantification of older people who have had a fall and who had multiple hospital admissions. Our results suggest that public health policy needs to target separate 
fall prevention messages and programs for males and females. The finding that most falls in older adults occur because of slips and trips supports the importance of community-based health services delivering fall prevention programs. Delivering appropriate fall prevention programs to special populations (such as people with neurological conditions) may help reduce hospital readmissions for falls. ${ }^{22}$ There is also a need for programs and strategies to prevent fall injury, particularly for residents of aged care facilities.

\section{Conclusion}

Rates of fall-related health service use continued to increase, despite the various health promotion and fall prevention strategies that were implemented in NSW during the study period. 2,25 Increased efforts and innovative strategies to prevent falls and associated injury among older people in NSW are needed, particularly safety and avoidance strategies targeted at males. Future linkage studies may provide a better understanding of the characteristics and overall healthcare usage of people who fall, and can assist in evaluating the effectiveness of fall prevention interventions.

\section{Acknowledgements}

This work is supported by the National Health and Medical Research Council (NHMRC) Partnerships for Better Health grant (ID: 1016876). The authors thank NSW Health for its in-kind support and contribution to the NHMRC Partnership Projects grant Widespread implementation of interventions to prevent falls in older people. Salary funding for SRL, LH, AT, LC and CS is provided by NHMRC fellowships.

\section{Competing interests}

None declared

\section{Author contributions}

SP was responsible for the study design, analysis of data, and drafting and editing of the manuscript. LH was responsible for drafting and editing, and contributing to data analysis. TC was responsible for acquiring the data, and reviewing and editing. QL was responsible for overseeing the data analysis, and reviewing and editing. SB was responsible for reviewing and editing, and contributing to the study design and data analysis. $\mathrm{AP}$ was responsible for acquiring the data, reviewing and editing, and contributing to the study design. AT, LC, SRL and JC were responsible for the study design, and reviewing and editing. SM was responsible for acquiring the data, and reviewing and editing. SL was responsible for the study design, reviewing and editing, and contributing to data analysis. CS was responsible for the study design, and drafting, reviewing and editing the manuscript.

\section{References}

1. Lord SR, Sherrington C, Menz HB, Close JCT. Falls in older people. 2nd ed. New York: Cambridge University Press; 2007.

2. NSW Ministry of Health. Snapshot November 2014: preventing falls and harm from falls. Sydney: NSW Ministry of Health; 2014 [cited 2017 Aug 30]. Available from: fallsnetwork.neura.edu.au/wp-content/ uploads/2014/12/NSWH6992_Snapshot_FallsPrevention_ final.pdf

3. Simpson PM, Bendall JC, Patterson J, Tiedemann A, Middleton PM, Close JC. Epidemiology of ambulance responses to older people who have fallen in New South Wales, Australia. Australas J Ageing. 2013;32(3):171-6.

4. Berry SD, Miller R. Falls: epidemiology, pathophysiology, and relationship to fracture. Curr Osteoporos Rep. 2008;6(4):149-54.

5. Kelsey JL, Procter-Gray E, Hannan MT, Li W. Heterogeneity of falls among older adults: implications for public health prevention. Am J Public Health. 2012;102(11):2149-56.

6. Watson WL, Clapperton AJ, Mitchell RJ. The cost of fallrelated injuries among older people in NSW, 2006-07. NSW Public Health Bull. 2011;22(3-4):55-9.

7. Harvey LA, Close JCT. Trends in fall-related hospitalisations, persons aged 65 years and older, NSW, 1998-99 to 2011-12. Sydney: Neuroscience Research Australia; 2013 [cited 2017 Aug 10]. Available from: fallsnetwork.neura.edu.au/wp-content/uploads/2011/03/ Trends-in-fall-related-hospitalisations-in-NSW-1998-99to-2011-12.pdf

8. Dowling AM, Finch CF. Baseline indicators for measuring progress in preventing falls injury in older people. Aust N Z J Public Health. 2009;33(5):413-7.

9. National Centre for Classification in Health. International statistical classification of diseases and related health problems, 10th revision, Australian modification. Sydney: University of Sydney; 2013.

10. University of Adelaide. Adelaide: Hugo Centre for Migration and Population Research; 2016. ARIA+2011; [cited 2015 Aug 30]. Available from: www.spatialonline. com.au/ARIA_2011/default.aspx

11. Australian Bureau of Statistics. Canberra: ABS, 2017. Statistics; 2015 [cited 2015 Feb 20]. Available from: www.abs.gov.au/AUSSTATS

12. Vu T, Finch CF, Day L. Patterns of comorbidity in community-dwelling older people hospitalised for fall-related injury: a cluster analysis. BMC Geriatr. 2011;11:45. 
13. Simpson P, Bendall J, Patterson J, Tiedemann A, Middleton P, Close J. The 'CATCH' study: a preliminary analysis describing the epidemiology of 500 prospectively collected cases involving older fallers attended to by paramedics. 2011 Paramedics Australasia Conference; 2011 Oct 7-8; Sydney, Australia. Australas J Paramed. 2013;10(2):26.

14. Simpson PM, Bendall JC, Tiedemann A, Lord SR, Close JC. Epidemiology of emergency medical service responses to older people who have fallen: a prospective cohort study. Prehosp Emerg Care. 2014;18(2):185-94

15. Thomas SL, Muscatello DJ, Middleton PM, Zheng W. Characteristics of fall-related injuries attended by an ambulance in Sydney, Australia: a surveillance summary. NSW Public Health Bull. 2011;22(3-4):49-54.

16. Mikolaizak AS, Simpson PM, Tiedemann A, Lord SR, Close JC. Systematic review of non-transportation rates and outcomes for older people who have fallen after ambulance service call-out. Australas J Ageing. 2013;32(3):147-57.

17. Mitra B, Cameron PA, Gabbe BJ. Ladders revisited. Med J Aust. 2007;186(1):31-4.

18. Nurmi I, Luthje P. Incidence and costs of falls and fall injuries among elderly in institutional care. Scand J Prim Health Care. 2002;20(2):118-22.
19. Rapp K, Becker C, Cameron ID, Konig HH, Buchele G. Epidemiology of falls in residential aged care: analysis of more than 70,000 falls from residents of Bavarian nursing homes. J Am Med Dir Assoc. 2012;13(2):187.e1-6.

20. Karmel R, Gibson D, Lloyd J, Anderson P. Transitions from hospital to residential aged care in Australia. Australas J Ageing. 2009;28(4):198-205.

21. Court-Brown CM, Clement ND, Duckworth AD, Aitken S, Biant LC, McQueen MM. The spectrum of fractures in the elderly. Bone Joint J. 2014;96-b(3):366-72.

22. Sherrington $C$, Michaleff ZA, Fairhall N, et al. Exercise to prevent falls in older adults: an updated systematic review and meta-analysis. Br J Sports Med. 2016; doi: 10.1136/bjsports-2016-096547. [Epub ahead of print].

23. Bleijlevens MH, Diederiks JP, Hendriks MR, van Haastregt JC, Crebolder HF, van Eijk JT. Relationship between location and activity in injurious falls: an exploratory study. BMC Geriatr. 2010;10:40.

24. Davie G, Langley J, Samaranayaka A, Wetherspoon ME. Accuracy of injury coding under ICD-10-AM for New Zealand public hospital discharges. Inj Prev. 2008; 14(5):319-23.

25. NSW Ministry of Health. Falls - prevention of falls and harm from falls among older people: 2011-2015. Policy directive. Sydney: NSW Government. Health; 2011 [cited 2017 Aug 10]. Available from: www1.health.nsw.gov.au/ pds/ActivePDSDocuments/PD2011_029.pdf

\section{Copyright: (c)}

(C) 2017 Paul et al. This article is licensed under the Creative Commons Attribution-NonCommercial-ShareAlike 4.0 International Licence, which allows others to redistribute, adapt and share this work non-commercially provided they attribute the work and any adapted version of it is distributed under the same Creative Commons licence terms. See: www.creativecommons.org/licenses/by-nc-sa/4.0/ 